\title{
Sleep deprivation selectively disrupts top-down adaptation to cognitive conflict in the Stroop test
}

\author{
WIM GEVERS ${ }^{1}$, GAETANE DELIENS ${ }^{1,2,3}$, SOPHIE HOFFMANN ${ }^{1}$, \\ WIM NOTEBAERT ${ }^{4}$ and PHILIPPE PEIGNEUX ${ }^{1,3}$ \\ ${ }^{1} \mathrm{CRCN}$ - Center for Research in Cognition \& Neurosciences and UNI - ULB Neurosciences Institute, Université Libre de Bruxelles (ULB), \\ Bruxelles, Belgium, ${ }^{2} \mathrm{CO} 3$, Consciousness, Cognition \& Computation Group at CRCN, Bruxelles, Belgium, ${ }^{3}$ UR2NF, Neuropsychology and \\ Functional Neuroimaging Research Group at CRCN, Bruxelles, Belgium and ${ }^{4}$ Department of Experimental Psychology, Ghent University, \\ Ghent,Belgium
}

\author{
Keywords \\ bottom-up process, cognitive adaptation, \\ sequential congruency effect, stroop \\ interference, top-down process \\ Correspondence \\ Prof. Wim Gevers, PhD, Center for Research in \\ Cognition and Neurosciences (CRCN), \\ Université Libre de Bruxelles (ULB), Campus du \\ Solbosch, CP191 Avenue F.D. Roosevelt 50, \\ B-1050 Bruxelles, Belgium. \\ Tel.: +32 265042 28; \\ fax: +32 265022 09; \\ e-mail: wim.gevers@ulb.ac.be
}

Accepted in revised form 23 May 2015; received 4 February 2015

DOI: $10.1111 /$ jsr.12320

\section{SUMMARY}

Sleep deprivation is known to exert detrimental effects on various cognitive domains, including attention, vigilance and working memory. Seemingly at odds with these findings, prior studies repeatedly failed to evidence an impact of prior sleep deprivation on cognitive interference in the Stroop test, a hallmark paradigm in the study of cognitive control abilities. The present study investigated further the effect of sleep deprivation on cognitive control using an adapted version of the Stroop test that allows to segregate top-down (attentional reconfiguration on incongruent items) and bottom-up (facilitated processing after repetitions in responses and/or features of stimuli) components of performance. Participants underwent a regular night of sleep or a night of total sleep deprivation before cognitive testing. Results disclosed that sleep deprivation selectively impairs top-down adaptation mechanisms: cognitive control no longer increased upon detection of response conflict at the preceding trial. In parallel, bottom-up abilities were found unaffected by sleep deprivation: beneficial effects of stimulus and response repetitions persisted. Changes in vigilance states due to sleep deprivation selectively impact on cognitive control in the Stroop test by affecting top-down, but not bottom-up, mechanisms that guide adaptive behaviours.

\section{INTRODUCTION}

Flexibility in information processing is a crucial requirement to perform adequately in an everyday environment. Flexibility and related processes are typically investigated using tasks in which participants must adapt to changing requirements (e.g. task-switching paradigms; Vandierendonck et al., 2010) or inhibit an automatic response in favour of another one in an interference context, as it is the case in the Stroop test (Stroop, 1935). Typically in the Stroop test, participants are instructed to report the ink colour of a colour word. The irrelevant colour word can be either congruent (e.g. the word 'blue' printed in blue) or incongruent (e.g. the word 'blue' printed in red). A consistent finding is that inhibitory processes involved in incongruent trials yield slower responses and more errors than congruent trials.

Sleep deprivation exerts a well-recognized negative impact on cognitive and brain processes subtending vigilance (Basner and Dinges, 2011) and other attentional functions
(Lo et al., 2012; Muto et al., 2012; Roca et al., 2012; Tomasi et al., 2009), with important inter-individual variability in vulnerability to sleep deprivation (Chee and Tan, 2010; Landolt, 2008). Sleep deprivation is also detrimental to working memory (Chee et al., 2006) and inhibition (Anderson and Platten, 2011) processes, but effects may be more variable (Chuah et al., 2006; Drummond et al., 2012) or much smaller and modulated by circadian phase (Lo et al., 2012) or time of day (Schmidt et al., 2012a). Notwithstanding, and perhaps surprisingly, available studies concur to suggest that interference effects in the Stroop task are actually not modulated by sleep deprivation. Indeed, studies conducted to date failed to evidence reduction of interference effects in this task, even after up to $40 \mathrm{~h}$ of extended wakefulness (Bratzke et al., 2012; Cain et al., 2011; Kim et al., 2011; Sagaspe et al., 2006).

However, trial-to-trial variations were not taken into account. Sequential congruency between successive trials modulates the Stroop interference effect (Notebaert and 
Verguts, 2007). That is, the interference effect for the current trial is much smaller after an incongruent (I) than after a congruent $(C)$ trial. This phenomenon, referred to as the congruency sequence effect (CSE), was initially explained in terms of top-down cognitive control mechanisms (Botvinick et al., 2001) when a conflict is detected, attentional reconfiguration takes place that increases the attentional resources to the relevant target dimension, thereby reducing the interference effect on the next trial. The CSE was found unchanged after sleep deprivation in a Flanker task (Tsai et al., 2005), suggesting the preservation of cognitive control mechanisms. However, a CSE can also be explained in terms of a bottom-up account: faster reaction times for trialto-trial congruency repetitions ( $\mathrm{I}-\mathrm{I}$ and $\mathrm{C}-\mathrm{C}$ ) as compared with congruency alternations (I-C and C-I; Mayr et al., 2003). Here, at least part of the CSE can be explained in terms of repetition of features of the stimulus and/or of the response (Mayr et al., 2003). Hence, both bottom-up and top-down factors may result in a so-called CSE in the Stroop task.

The impact of sleep deprivation was examined, respectively, on bottom-up and top-down factors in the Stroop test. The task had three possible stimuli and responses to allow both trial-to-trial repetition and alternation in responses and/ or stimuli features (Notebaert et al., 2006), and data were analysed using a two-step multiple regression analysis (Notebaert and Verguts, 2007). In a first step, the regression implements bottom-up predictors that reliably predict reaction times. In a second step, residual variance predicts topdown adaptation on top of bottom-up effects. A change in the CSE after sleeping compared with after sleep deprivation would be revealed as an interaction between Condition (sleep or sleep deprivation) and the respective bottom-up or top-down predictors.

Hence, Stroop performance can be decomposed into three levels. The first level is the size of the interference effect, which depends on how successfully the participants can ignore the irrelevant information (e.g. selective attention). The second level is bottom-up sequential modulation, which encompasses the beneficial effects of stimulus and response repetitions. And finally, the third level is top-down sequential modulation, reflecting cognitive control adjustments. The current experiment aimed at determining whether one or several of these processing levels subtending performance in the Stroop task suffer from sleep deprivation.

\section{MATERIALS AND METHODS}

\section{Participants}

Participants were recruited through word of mouth and advertisements at the faculty of psychology. Twenty-five healthy subjects (five males; mean age $\pm \mathrm{SD}=21.12 \pm 1.88$ years) participated for monetary reward in this experiment. The larger number of females reflects the gender distribution in the faculty of psychology. Three subjects were removed from the statistical analyses because of bad performance during the regular sleep (RS) condition (see later), and three additional participants were removed due to non-optimal sleep quality during the past month (Pittsburgh Sleep Quality Index > 6; Buysse et al., 1989). The 19 remaining participants (five males; mean age $\pm S D=20.95 \pm 1.96$ years) were French-speaking, right-hand dominant [on a range from -100 (fully left handed) to +100 (fully right handed), average score $75.79 \pm 44.26$; Edinburgh handedness questionnaire; Oldfield, 1971], with normal or corrected-to-normal vision and no prior history of sleep disorders. Six were moderate evening types, 10 intermediate types, two moderate morning types, and one reached the cutoff for extreme morning type (Morningness-Eveningness Questionnaire; Horne and Ostberg, 1976; range 36-72). Participants gave written, informed consent to participate in this study approved by the local Ethics committee of the Université Libre de Bruxelles (ULB). They were required to keep regular sleep patterns during the week before and throughout the experiment, and to avoid napping on experimental days. To control for the regularity of sleep habits, they were asked to complete daily sleep logs (St Mary's Hospital sleep questionnaire; Ellis et al., 1981) for the 2 days before each testing condition.

\section{Materials and procedure}

Participants were tested on computers running E-PRIME 2.0 software (Psychology Software Tools, Pittsburgh, PA, USA). Stimuli consisted of the French colour word names 'VERT' (green), 'JAUNE' (yellow) and 'ROUGE' (red) displayed in Arial capital fonts in the possible ink colours green, yellow and red. Words were approximately $2 \mathrm{~cm}$ wide and $0.5 \mathrm{~cm}$ high. Participants had to react as fast as possible by indicating the stimulus ink colour using the index, middle or ring finger of their dominant (right) hand to press the 'c', ' $v$ ', or 'b' keys on a standard Belgian-French keyboard. Stimuli were randomized per participant and presented in the centre of the computer's screen approximately $50 \mathrm{~cm}$ from the participants' eye, and remained on display until a response key was pressed, after which a grey fixation cross was presented for $750 \mathrm{~ms}$, followed by the next stimulus. The nine possible word-ink colour combinations were presented in random order, resulting in 33\% congruent trials and $66 \%$ incongruent trials. For $33 \%$ of the trials, the irrelevant word was repeated, and for another $33 \%$ the relevant colour (response repetitions) was repeated. No repetition at all was present in the remaining 33\%. Participants were first presented a practice block of 90 trials with feedback, followed by eight consecutive blocks of 90 trials without feedback (total 720 experimental trials).

A within-subjects design was used. For all participants, the Stroop task was administered twice at 09:00 hours, once after a night of sleep (RS) and once after a night of total sleep deprivation (SD condition). Conditions were administered in a counterbalanced order between participants and spaced apart by 1 week at least. During the RS night, participants slept normally at home. During the SD night, they were kept 
in the laboratory from $22: 00$ hours to $10: 00$ hours under supervision of one of the experimenters. During the SD night, participants were kept in a dimmed light room (<10 lux) and isocaloric snacks were provided hourly with continuous free water access. Participants were asked to engage in quiet activities (e.g. reading, watching movies) during the night. The Karolinska Sleepiness Scale (KSS; Akerstedt and Gillberg, 1990) was administered hourly during the SD night. Smoking, alcohol and stimulant drinks were not allowed on experimental days (including the day where the SD started) and in the SD night.

\section{Data analysis: multiple regression}

Statistical analyses were conducted using sPSs software version 20.0 (IBM, Armonk, NY, USA). Data were analysed using the multiple regression approach outlined in the Introduction (for a detailed presentation, see Notebaert and Verguts, 2007; see also Lorch and Myers, 1990). Briefly, bottom-up predictors are taken into account in a first step. In the second step, new analyses are performed on the residual variance to test whether conflict adaptation can explain the variance that was not accounted for by bottomup effects.

In the first step, besides Congruency effects (congruent or incongruent) and Condition (RS or SD), five binary bottomup factors were selected as well as their interaction with Condition. These are the factors that can explain the CSE in terms of bottom-up feature repetition and integration effects. The interaction of these factors with Condition informs us about the influence of sleep deprivation on these bottom-up factors. The first two factors were: (i) repetition of the target colour (sequence of relevant information: target is repeated); and (ii) repetition of the distractor word (sequence of irrelevant information: distractor is repeated). Other factors encompassed: (iii) feature integration [complete (target and distractor repeated) versus partial (target or distractor repeated) repetitions and alternations (no repetition)]; and (iv) negative priming (i.e. distractor becomes target). Only transitions where both $n-1$ and $n$ were incongruent were regarded as negative priming. The final bottom-up factor (v) was defined by the transitions where relevant information on the previous trial became the irrelevant factor on the next trial (Rel-Irrel; target of the previous trial becomes the distractor on the current trial). The second step tested whether top-down factors could explain the residual variance from the first step. To test for conflict adaptation, a factor with the value of 1 was entered if congruency was repeated, and the value of 0 if congruency was alternated. Also, the congruency of the previous trial was included because lower error rates and a trend towards slower response times following incongruent trials were observed in a prior study (Ullsperger et al., 2005). Finally, the interactions of both factors with Condition were entered to investigate whether sleep deprivation had a selective influence on the top-down factors or not.

\section{RESULTS}

\section{Sleep and vigilance parameters}

The mean sleep duration for the penultimate night before the experimental manipulation $(N-2)$ was $8 \mathrm{~h} 24 \mathrm{~min}$ (SD $\pm 58 \mathrm{~min}$, range $6 \mathrm{~h} 07 \mathrm{~min}-10 \mathrm{~h} 30 \mathrm{~min}$ ) in the $\mathrm{RS}$ and $8 \mathrm{~h} 33 \mathrm{~min}$ ( $\pm 1 \mathrm{~h} 33 \mathrm{~min}$, range $5 \mathrm{~h} 30 \mathrm{~min}-12 \mathrm{~h}$ ) in the SD condition. The mean sleep duration for the night before the experimental manipulation $(N-1)$ was $7 \mathrm{~h}$ $18 \mathrm{~min}$ (SD $\pm 1 \mathrm{~h} 15 \mathrm{~min}$, range $5 \mathrm{~h} 30 \mathrm{~min}-10 \mathrm{~h}$ ) in the $\mathrm{RS}$ and $8 \mathrm{~h} 31 \mathrm{~min}$ ( $\pm 1 \mathrm{~h} 07 \mathrm{~min}$, range $6 \mathrm{~h} 20 \mathrm{~min}-11 \mathrm{~h}$ ) in the SD condition. A repeated-measure ANOVA conducted on mean sleep duration with within-subject factors Night ( $N-2, N-1$ before testing) and Condition (RS versus SD) and between-subject factor Order of the conditions (SD-RS versus RS-SD) disclosed a main effect of Condition $\left(F_{1,17}=10.51 ; P=0.005\right)$, with longer sleep duration in the sleep deprivation condition than in the sleep condition and a trend for a main effect of Night of sleep $\left(F_{1,17}=4.17 ; P=0.06\right)$ with longer sleep duration at night $N-2$ than at night $N-1$. The interaction between Night and Condition was also significant $\left(F_{1,17}=4.70 ; P=0.04\right)$. Post hoc tests indicate that the average sleep duration for the night before the experimental manipulation $(N-1)$ in the RS condition was marginally shorter than at night $N-2(P=0.06)$, and significantly shorter than at nights $N-1(P=0.03)$ and $N-2(P=0.03)$ in the SD condition. All other interactions or effects were not significant (all $P>0.28)$.

As expected during the SD night, KSS scores steadily increased over successive hours [from well awake at 22:00 hours $(2.53 \pm 1.71)$ to very tired at 09:00 hours $\left.(6.74 \pm 1.82) ; \quad F_{11,198}=32.19 ; P=0.000\right]$. Vigilance state was assessed in the two conditions by using the overall variance in RTs in the Stroop task, for which the coefficient of variance $(\mathrm{CV}=\mathrm{Stdev} / \mathrm{avr} \mathrm{RT})$ was calculated. Across participants, the $\mathrm{CV}$ was 0.34 for the sleep condition and 0.47 for the sleep deprivation condition $\left(t_{18}=-5.33 ; P<0.0001\right)$, suggesting increased general state instability after sleep deprivation.

\section{Multiple regression for Stroop performance in RS and SD conditions}

Excessively slow responses or high number of errors can be the consequence of SD in itself. For this reason, data trimming was performed on the basis of the RS condition only. Participants falling out the RS condition were also eliminated from the SD condition. This was the case for three participants exhibiting excessively slow responses (>2 SD from the mean; $n=1$ ) or too many errors ( $>2$ SD from the mean, $n=2)$. Errors and trials following an error $(8.89 \%$ of trials) were excluded from the analyses on RTs. Reaction times faster than $200 \mathrm{~ms}$ (commissions) and slower than $3000 \mathrm{~ms}$ were also excluded (1.14\% of trials). 
In addition to the regression analysis, Fig. 1 shows CSEs on the basis of the mean reaction times in all conditions (RS or SD and bottom-up or top-down). A first analysis demonstrated that the order of tasks (e.g. first RS or first SD) did not interact with any of the factors of interest. It was therefore not taken into consideration for the remaining analyses.

Results of the regression analysis are reported in Table 1. Regression parameters (i.e. beta) refer to the unique contribution of each predictor on the mean RT. The regression parameter or value of each predictor should be interpreted as the unique effect of this predictor on the mean RT. For instance, a value of 50 indicates that when this factor takes on the value of 1 , RTs are on average $50 \mathrm{~ms}$ slower than when this factor takes on the value of 0 , when all other factors are held constant. As shown in Table 1, results disclosed a main effect for Stroop congruency. To illustrate the reading of Table 1 (top rows), participants were about $29 \mathrm{~ms}$ faster on congruent compared with incongruent trials $(P<0.001)$. The effect of Condition demonstrated a general slowing after sleep deprivation $(P<0.001)$. This analysis also highlighted contributions of target sequence and feature integration factors in both conditions $(P<0.05)$. Interestingly, also the factor testing for the interaction between Condition and Congruency was significant $(P<0.05)$, showing that the congruency effect was $18 \mathrm{~ms}$ stronger after SD than after RS. An additional analysis demonstrated that this stronger congruency was not due to mental fatigue induced by the length of the experiment: regardless of the condition, the Stroop congruency effect was equally strong in the first as in the second half of the experiment $(F<1)$.

The second step of the analysis investigated possible topdown adaptation. Importantly, the results of the second step analysis (Table 1, bottom rows) revealed a significant interaction between conflict adaptation and Condition, indicating stronger conflict adaptation after RS than after $\mathrm{SD}(P<0.05)$. None of the other factors reached significance (all $P>0.15$ ). Hence, the analysis indicates that even when bottom-up factors are taken into account, top-down conflict adaptation still influenced RTs in the RS but not in the SD condition.

\section{DISCUSSION}

The aim of the present study was to investigate the influence of sleep deprivation on the different components subtending interference effects in the Stroop task. The results demonstrate that top-down cognitive control mechanisms are altered by sleep deprivation, whereas bottom-up mechanisms remain essentially unchanged. Indeed, feature repetition effects were robustly observed both after a normal night of sleep and after a night of sleep deprivation. Top-down conflict adaptation, however, was only observed after a night of sleep.

Bottom-up sequential modulation is usually interpreted in terms of simple priming mechanisms, where for instance the presentation of the same stimulus primes the execution of the same response, thus resulting in faster RTs (Mayr et al., 2003). Top-down conflict adaptation, on the other hand, relies on a widespread control network that necessitates the availability of cognitive resources (Botvinick et al., 2001). According to the latter theory, conflict adaptation is the readjustment of selective attention with more attention to relevant information after conflict detection. Sleep deprivation is known to exert a deleterious effect on various attentional parameters (see, for reviews, Lim and Dinges, 2010a;
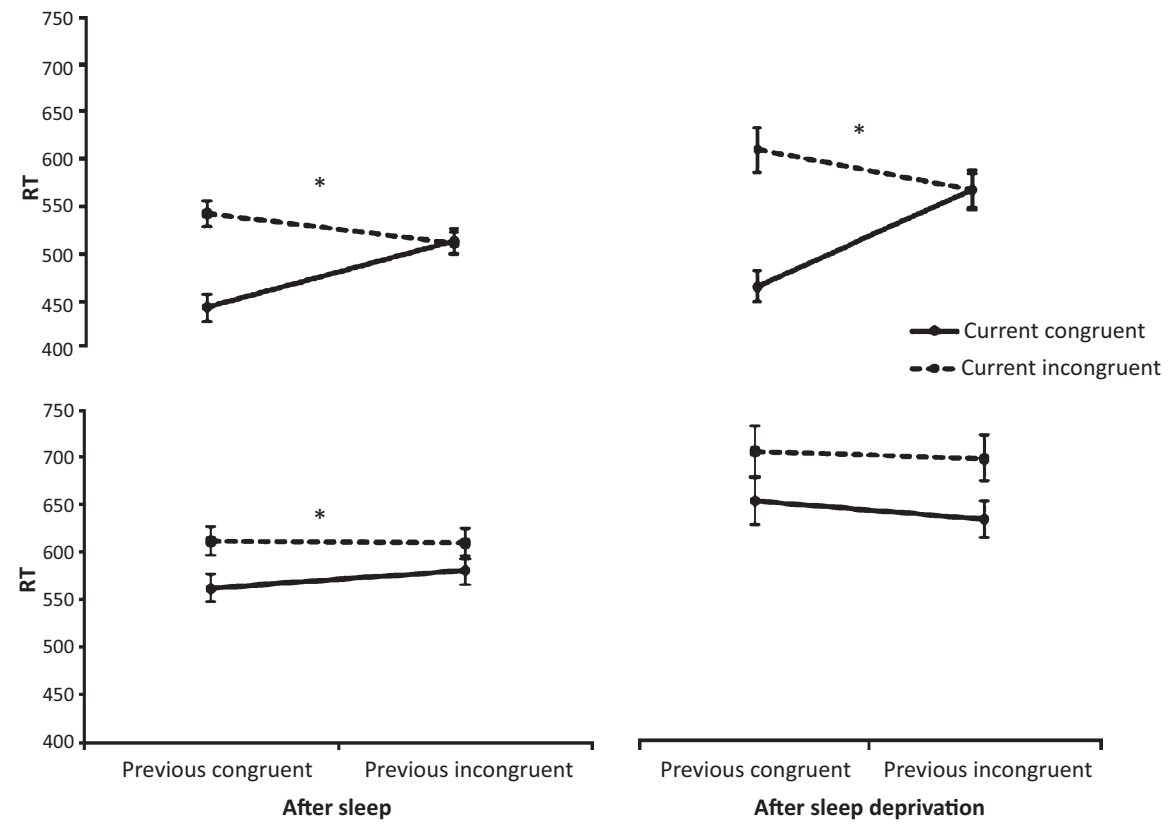

Previous congruent Previous incongruent

After sleep deprivation

Figure 1. Interactions between previous and current congruency effects both after sleep and after sleep deprivation. The top panels indicate the congruency sequence effect (CSE) as a function of bottom-up factors. The lower panel indicates the CSE as a function of top-down factors. Significant CSEs are indicated with $(*)$. Error bars indicate one SEM. 
Table 1 Results of two-step multiple regression analysis both after normal sleep and after sleep deprivation Predictors under the line were added in step two of the analysis. Average beta values are presented together with their standard errors

\begin{tabular}{lccc}
\hline & & Stroop performance \\
\cline { 2 - 3 } & $\beta$ (avr across participants) & One sample t-test t(18) & \\
\hline Predictor & & & \\
Condition (RS or SD) & $94,62(21,37)$ & $-6,428$ & 0 \\
Congruency & $-29,26(4,39)$ & $-2,668$ & 0 \\
Condition $\times$ Congruency & $-18,45(6,93)$ & $-7,900$ & 0.016 \\
Sequence of targets & $-115,54(14,63)$ & $-1,851$ & 0 \\
Condition $\times$ Sequence of targets & $-24,79(13,39)$ & -0.884 & 0.081 \\
Sequence of distractor & $-3,18(5,2)$ & 0.277 & 0.388 \\
Condition $\times$ Sequence of distractor & $-4,79(5,42)$ & $-2,503$ & 0.785 \\
Feature integration & $-18,23(7,28)$ & $-1,255$ & 0.022 \\
Condition $\times$ feature integration & $-16,18(18)$ & -0.135 & 0.225 \\
Target becomes distractor & $-0,39(2,92)$ & -0.151 & 0.909 \\
Condition $\times$ target becomes distractor & $-0,99(6,56)$ & $-1,092$ & 0.894 \\
Distractor becomes target & $7,33(8,06)$ & 1.296 \\
Condition $\times$ distractor becomes target & $-16,99(15,55)$ & -1.138 & 0.882 \\
Congruency of previous trial & $3,59(2,77)$ & -1.500 & 0.375 \\
Condition $\times$ congruency of previous trial & $-4,10(3,61)$ & 2.634 \\
Conflict adaptation & $-3,52(2.34)$ & 0.289 \\
Condition $\times$ conflict adaptation & $6,60(2,50)$ & 0.211 \\
\hline
\end{tabular}

Schmidt et al., 2007). Therefore, both the overall size of the Stroop effect (selective attention) and the sequential modulation of the congruency effect (reset of selection attention) could be affected by sleep deprivation. Complementary to this interpretation, Verguts and Notebaert (2009) linked conflict adaptation to arousal, proposing that the experienced conflict during incongruent trials triggers a small arousal response that facilitates binding between task-relevant cortical areas. Such adaptation-by-binding account might explain the effects of sleep deprivation in terms of reduced arousal, in line with neuroimaging studies showing that both circadian and homeostatic parameters have an impact on both arousal and higher-level cognitive parameters (Schmidt et al., 2009, 2012b). In line with the current results, Horowitz et al. (2003) observed that sleep deprivation did not affect shifts of attention in a visual search task, but that less topdown control was exerted at the decision level. Others found that sleep deprivation actually exerts an impact on all components of the attentional network (Muto et al., 2012; but see Roca et al., 2012), which makes it difficult at this point to disentangle these complementary accounts.

Besides the global preservation of bottom-up effects and the elimination of top-down influences after sleep deprivation, the regression analysis also yielded evidence for a stronger Stroop congruency effect after sleep deprivation than after regular sleep. This observation is in contrast with prior studies reporting no influence of sleep deprivation on the Stroop interference (Bratzke et al., 2012; Cain et al., 2011; Sagaspe et al., 2006). Nevertheless, some methodological differences exist between the current and other studies. In Sagaspe et al. (2006), participants had to respond verbally rather than manually, which might change the dynamics of the Stroop task. Other studies used proportionally more incongruent than congruent trials (Cain et al., 2011), or congruent trials were not included (i.e. only incongruent and neutral trials in Sagaspe et al., 2006). Amongst prior studies, the design used by Bratzke et al. (2012) is probably the most similar to the current one. Participants used manual responses with four different colours, and were presented the same amount of congruent and incongruent trials. At variance with the current study, however, participants were tested numerous times. Whereas the Stroop task was only administered twice in the current study (once with, once without sleep deprivation), it was administered multiple times within a single participant in prior studies using constant routine protocols (Bratzke et al., 2012; Cain et al., 2011; Sagaspe et al., 2006). Although these protocols allow more efficiently disentangling the effects of accumulated sleep pressure from those of circadian position (Schmidt et al., 2007), multiple repetitions of a task pertaining to the executive functions domain may favour the development of alternate strategies (Blatter et al., 2005) and/or introduce a trade-off between practice and sleep deprivation. This tradeoff would indicate that participants developed to some extent a 'reading suppression response' (Dulaney and Rogers, 1994; Feinstein et al., 1994) through task repetition, which may counteract the deleterious effect of sleep deprivation. Whether these differences are sufficient to explain the fact that the current study observed a subtle modulation of the Stroop congruency effect while others did not, remains at this point an empirical question that should be investigated in further studies.

In the present study, it was unambiguously demonstrated that sleep deprivation exerts a selective detrimental impact on selective attention and cognitive control. Additionally, an increased Stroop interference effect after sleep deprivation was observed, a phenomenon indicative of impaired selective 
attention mechanisms. In line with this interpretation, Lim and colleagues (Lim et al., 2010b) demonstrated using functional magnetic resonance imaging a negative influence of sleep deprivation on target detection abilities, with attention-driven biasing of information processing after normal sleep but not after sleep deprivation. Similarly, Trujillo et al. (2009) found that 1 night of sleep deprivation impacts endogenously driven selective attention (top-down) more than exogenously driven (bottom-up) selective attention. In this respect, one could argue that sustaining a focus on the task-relevant dimension in the Stroop task also requires to some extent endogenous attention. An alternative or complementary explanation would be that automatic bottom-up effects become more prominent as top-down cognitive control processes no longer inhibit them. Accordingly, performance for automatic memory processes is better when participants are tested at their non-preferred time of the day (Delpouve et al., 2014; May et al., 2005), i.e. when cognitive resources are less available. At present, the current results show that changes in vigilance states due to sleep deprivation selectively impact on cognitive control in the Stroop test by affecting the top-down, but not bottom-up, mechanisms that guide adaptive behaviours.

\section{ACKNOWLEDGEMENTS}

Dr Gaétane Deliens was Research Fellow of the FRS-FNRS Fonds National de la Recherche Scientifique, Belgium during the study. The authors thank the volunteers for participating in the study.

\section{AUTHOR CONTRIBUTIONS}

GD was Research Fellow at the FRS-FNRS Fonds National de la Recherche Scientifique, Belgium.

\section{CONFLICT OF INTEREST}

None of the authors has a potential conflict of interest.

\section{REFERENCES}

Akerstedt, T. and Gillberg, M. Subjective and objective sleepiness in the active individual. Int. J. Neurosci., 1990, 52: 29-37.

Anderson, C. and Platten, C. R. Sleep deprivation lowers inhibition and enhances impulsivity to negative stimuli. Behav. Brain Res., 2011, 217: 463-466.

Basner, M. and Dinges, D. F. Maximizing sensitivity of the psychomotor vigilance test (PVT) to sleep loss. Sleep, 2011, 34: 581-591.

Blatter, K., Opwis, K., Munch, M., Wirz-Justice, A. and Cajochen, C. Sleep loss-related decrements in planning performance in healthy elderly depend on task difficulty. J. Sleep Res., 2005, 14: 409-417.

Botvinick, M. M., Braver, T. S., Barch, D. M., Carter, C. S. and Cohen, J. D. Conflict monitoring and cognitive control. Psychol. Rev., 2001, 108: 624.

Bratzke, D., Steinborn, M. B., Rolke, B. and Ulrich, R. Effects of sleep loss and circadian rhythm on executive inhibitory control in the Stroop and Simon tasks. Chronobiol. Int., 2012, 29: 55-61.
Buysse, D. J., Reynolds, C. F. R., Monk, T. H., Berman, S. R. and Kupfer, D. J. The Pittsburgh Sleep Quality Index: a new instrument for psychiatric practice and research. Psychiatry Res., 1989, 28: 193-213.

Cain, S. W., Silva, E. J., Chang, A. M., Ronda, J. M. and Duffy, J. F. One night of sleep deprivation affects reaction time, but not interference or facilitation in a Stroop task. Brain Cogn., 2011, 76: $37-42$.

Chee, M. W. and Tan, J. C. Lapsing when sleep deprived: neural activation characteristics of resistant and vulnerable individuals. Neurolmage, 2010, 51: 835-843.

Chee, M. W., Chuah, L. Y., Venkatraman, V., Chan, W. Y., Philip, P. and Dinges, D. F. Functional imaging of working memory following normal sleep and after 24 and $35 \mathrm{~h}$ of sleep deprivation: correlations of fronto-parietal activation with performance. Neurolmage, 2006, 31: 419-428.

Chuah, Y. M., Venkatraman, V., Dinges, D. F. and Chee, M. W. The neural basis of interindividual variability in inhibitory efficiency after sleep deprivation. J. Neurosci., 2006, 26: 7156-7162.

Delpouve, J., Schmitz, R. and Peigneux, P. Implicit learning is better at subjectively defined non-optimal time of day. Cortex, 2014, 58: $18-22$.

Drummond, S. P., Anderson, D. E., Straus, L. D., Vogel, E. K. and Perez, V. B. The effects of two types of sleep deprivation on visual working memory capacity and filtering efficiency. PLOS ONE, 2012, 7: e35653.

Dulaney, C. L. and Rogers, W. A. Mechanisms underlying reduction in Stroop interference with practice for young and old adults. J. Exp. Psychol., 1994, 20: 470-484.

Ellis, B. W., Johns, M. W., Lancaster, R., Raptopoulos, P., Angelopoulos, N. and Priest, R. G. The St. Mary's Hospital sleep questionnaire: a study of reliability. Sleep, 1981, 4: 93-97.

Feinstein, A., Brown, R. and Ron, M. Effects of practice of serial tests of attention in healthy subjects. J. Clin. Exp. Neuropsychol., 1994, 16: $436-447$.

Horne, J. A. and Ostberg, O. A self-assessment questionnaire to determine morningness-eveningness in human circadian rhythms. Int. J. Chronobiol., 1976, 4: 97-110.

Horowitz, T. S., Cade, B. E., Wolfe, J. M. and Czeisler, C. A. Searching night and day: a dissociation of effects of circadian phase and time awake on visual selective attention and vigilance. Psych. Sci., 2003, 14: 549-557.

Kim, H. J., Kim, J. H., Park, K. D., Choi, K. G. and Lee, H. W. A survey of sleep deprivation patterns and their effects on cognitive functions of residents and interns in Korea. Sleep Med., 2011, 12 : 390-396.

Landolt, H. P. Genotype-dependent differences in sleep, vigilance, and response to stimulants. Curr. Pharm. Des., 2008, 14: 33963407.

Lim, J. and Dinges, D. F. A meta-analysis of the impact of short-term sleep deprivation on cognitive variables. Psychol. Bull., 2010a, 136: 375-389.

Lim, J., Tan, J. C., Parimal, S., Dinges, D. F. and Chee, M. W. Sleep deprivation impairs object-selective attention: a view from the ventral visual cortex. PLOS ONE, 2010b, 5: e9087.

Lo, J. C., Groeger, J. A., Santhi, N. et al. Effects of partial and acute total sleep deprivation on performance across cognitive domains, individuals and circadian phase. PLOS ONE, 2012, 7: e45987.

Lorch, R. F. and Myers, J. L. Regression analyses of repeated measures data in cognition research. J. Exp. Psychol., 1990, 16: 149-157.

May, C. P., Hasher, L. and Foong, N. Implicit memory, age, and time of day: paradoxical priming effects. Psychol. Sci., 2005, 16: 96-100.

Mayr, U., Awh, E. and Laurey, P. Conflict adaptation effects in the absence of executive control. Nat. Neurosci., 2003, 6: 450-452.

Muto, V., Shaffii-Le Bourdiec, A., Matarazzo, L. et al. Influence of acute sleep loss on the neural correlates of alerting, orientating 
and executive attention components. J. Sleep Res., 2012, 21: 648658.

Notebaert, W. and Verguts, T. Dissociating conflict adaptation from feature integration: a multiple regression approach. J. Exp. Psychol., 2007, 33: 1256-1260.

Notebaert, W., Gevers, W., Verbruggen, F. and Liefooghe, B. Topdown and bottom-up sequential modulations of congruency effects. Psychon. Bull. Rev., 2006, 13: 112-117.

Oldfield, R. C. The assessment and analysis of handedness: the Edinburgh inventory. Neuropsychologia, 1971, 9: 97-113.

Roca, J., Fuentes, L. J., Marotta, A. et al. The effects of sleep deprivation on the attentional functions and vigilance. Acta Psychol., 2012, 140: 164-176.

Sagaspe, P., Sanchez-Ortuno, M., Charles, A. et al. Effects of sleep deprivation on Color-Word, Emotional, and Specific Stroop interference and on self-reported anxiety. Brain Cogn., 2006, 60: 76-87.

Schmidt, C., Collette, F., Cajochen, C. and Peigneux, P. A time to think: circadian rhythms in human cognition. Cogn. Neuropsychol., 2007, 24: 755-789.

Schmidt, C., Collette, F., Leclercq, Y. et al. Homeostatic sleep pressure and responses to sustained attention in the suprachiasmatic area. Science, 2009, 324: 516-519.

Schmidt, C., Peigneux, P., Cajochen, C. and Collette, F. Adapting test timing to the sleep-wake schedule: effects on diurnal neuro- behavioral performance changes in young evening and older morning chronotypes. Chronobiol. Int., 2012a, 29: 482-490.

Schmidt, C., Peigneux, P., Leclercq, Y. et al. Circadian preference modulates the neural substrate of conflict processing across the day. PLoS ONE, 2012b, 7: e29658.

Stroop, J. R. Studies of interference in serial verbal reactions. J. Exp. Psychol., 1935, 18: 643.

Tomasi, D., Wang, R. L., Telang, F. et al. Impairment of attentional networks after 1 night of sleep deprivation. Cereb. Cortex, 2009, 19: 233-240.

Trujillo, L. T., Kornguth, S. and Schnyer, D. M. An ERP examination of the different effects of sleep deprivation on exogenously cued and endogenously cued attention. Sleep, 2009, 32: 1285-1297.

Tsai, L. L., Young, H. Y., Hsieh, S. and Lee, C. S. Impairment of error monitoring following sleep deprivation. Sleep, 2005, 28: 707-713.

Ullsperger, M., Bylsma, L. M. and Botvinick, M. M. The conflict adaptation effect: it's not just priming. Cogn. Affect. Behav. Neurosci., 2005, 5: 467-472.

Vandierendonck, A., Liefooghe, B. and Verbruggen, F. Task switching: interplay of reconfiguration and interference control. Psychol. Bull., 2010, 136: 601-626.

Verguts, T. and Notebaert, W. Adaptation by binding: a learning account of cognitive control. Trends Cogn. Sci., 2009, 13: 252257. 